\title{
Arrhythmogenic right ventricular cardiomyopathy associated ventricular tachycardia misdiagnosed as idiopathic right ventricular outflow tract origin and its management
}

\author{
Xiangmin Shi', Yutang Wang, Zhaoliang Shan \\ Department of Cardiology, The General Hospital of People’s Liberation Army, Beijing, China; \\ *Corresponding Author: shixm301cardiac@hotmail.com
}

Received 25 June 2013; revised 23 July 2013; accepted 20 August 2013

Copyright (C) 2013 Xiangmin Shi et al. This is an open access article distributed under the Creative Commons Attribution License, which permits unrestricted use, distribution, and reproduction in any medium, provided the original work is properly cited.

\begin{abstract}
Arrhythmogenic right ventricular cardiomyopathyldysplasia (ARVC/D) is an autosomal dominant genetic form of cardiomyopathy (CM), which primarily affects the right ventricle (RV) and results in life threatening ventricular arrhythmias and sudden cardiac death (SCD). Diagnosis is difficult due to the broad spectrum of phenoltypic variations, especially in the early stage. Clinical suspicion should be raised in the setting of refractory ventricular tachycardia originating from the RV, and the final diagnosis could be made based on the combination of electrocardiography, echocardiography, cardiac magnetic resonance imaging (CMRI) and myocardial biopsy. Implantable cardioverter-defibrillator (ICD) implantation is an effective option for the treatment of ARVC.
\end{abstract}

Keywords: Arrhythmogenic Right Ventricular Cardiomyopathy; Ventricular Tachycardia; Cardiac Magnetic Resonance Imaging

\section{INTRODUCTION}

Arrhythmogenic right ventricular cardiomyopathy (ARVC) is one of the major causes of recurrent ventricular tachycardia (VT) and sudden cardiac death in young people. QRS morphology of VT and abnormalities of precordial leads in sinus rhythm, in association with echocardiography (UCG) and cardiac magnetic resonance imaging (CMR), play an important role in early diagnosis of ARVC. Presented herein is a 25-year-old male soldier who suffered from recurrent episodes of palpitation and presyncope due to VT, which resembled that of idio- pathic right ventricular outflow tract (RVOT) origin, diagnosed as idiopathic RVOT VT in the presence of echocardiographic normal heart and received oral administration of metoprolol which eventually proved to be ineffective. Meanwhile, surface 12 leads ECG indicated persistent $\mathrm{T}$ wave inversion in $\mathrm{V} 1-\mathrm{V} 4$, which was suggestive of ARVC, and following CMR scan confirmed the diagnosis, and subsequently the patient was treated with implantable cardioverter defibrillator (ICD).

\section{CASE REPORT}

A 25-year old male soldier without significant structural heart disease and past medical therapy as well as family history of sudden death, complained of recent onset of recurrent palpitation and presyncope. Documented ECG in local clinic revealed VT of left bundle branch block (LBBB) morthology with a inferior axis (Figure 1(b)), which was similar to VT arising from RVOT. Physical examination and routine laboratory tests, including ESR, CRP, cardiac-specific enzymes, D-dimer and serum electrolytes, were within normal limits. ECG indicated Rs pattern of QRS complex of normal width in lead V1-V3 and T wave inversion in V1-V4 (Figure 1(a)), no epsilon wave was seen. Firstly ARVC was suspected, however, UCG ruled out the possibility in the setting of structurally normal left and right ventricular. The patient was primarily diagnosed as idiopathic RVOT-VT and recommended the radiofrequency ablation therapy, which was declined by him latterly. In the following several weeks, oral administration of metoprolol (25 mg, bid) was prescribed to prevent the occurrence of ventricular arrhythmia, however, he still complained frequent episode of palpitation with presyncope, and consequently refered for hospitalization for further examination.

During hospitalization, repeated trans-thoracic UCG 


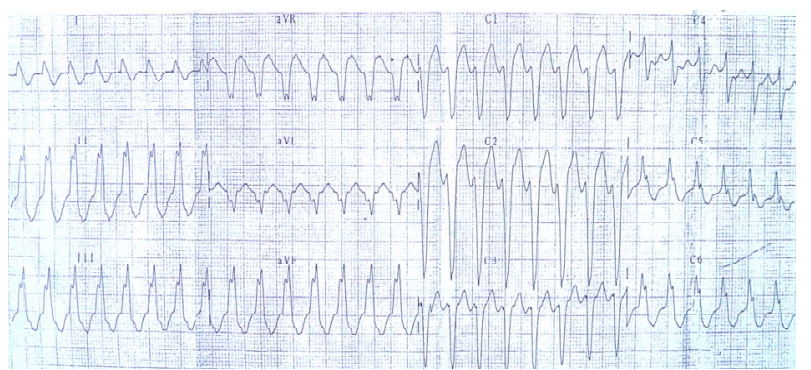

(a)

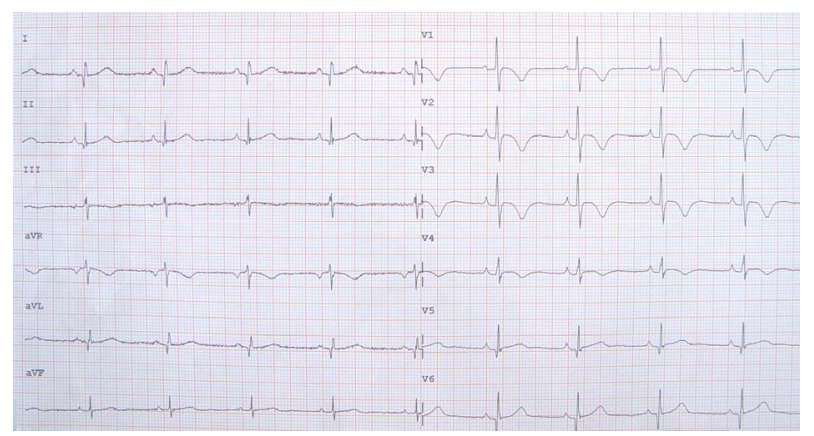

(b)

Figure 1. (a) ECG of sinus rhythm revealing $T$ wave inversion in lead V1-V4; (b) ECG documented ventricular tachycardia with LBBB morphology and a inferior axis similar to idiopathic RVOT-VT.

revealed normal dimension, thickness and ejection fraction of dual ventricular chamber. He was still suspected the diagnosis of ARVC due to the surface ECG findings, Gadolinium-enhanced cardiac MRI (CMR) scan was subsequently arranged for detailed assessment, which revealed abnormality of right ventricular including focal enlargement, akinetic motion with aneurysm of the free wall (Figure 2), these findings were strongly suggestive of ARVC.

Based on those clinical features, the soldier fulfilled the clinical criteria for ARVC and ICD was implanted consequently, during the follow-up, we observed ICD treatment in the presence of VT, including anti-tachycardia pacing (ATP) and shock delivery, the patient no longer suffered presyncope.

\section{DISCUSSION}

ARVC is characterized by the progressive replacement of right ventricular myocytes by fibrous or fibro-fatty tissue which results in an area of slow conduction and dispersion of refractoriness [1], leading to reentrant ventricular tachyarrhythmia. Mutations in the desmosomal proteins of the myocardium are believed to be attributable to the pathogenesis of ARVC. However, the diagnosis of ARVC is challenging due to its wide range of manifestations ranging from an absence of symptoms to cardiac arrest. Currently the 1994 Task Force Criteria
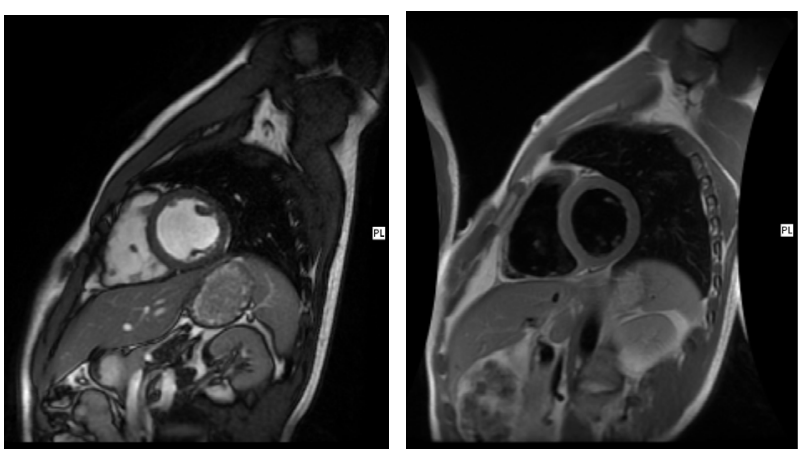

Figure 2. CMR scan indicating focal enlargement, akinetic motion with aneurysm (arrow indicated) localized in the free wall of right ventricular. Left: enhenced-CMR imaging; right: non enhenced-CMR imaging.

(TFC) has been the frequently used method for the diagnosis of ARVC based on electrocardiographic, structural, functional abnormalities and family history [2].

The patient firstly referred to our hospital with recurrent VT of LBBB morphology and an inferior axis, presenting as an R wave in II, III and AVF leads; QS wave in AVR and AVR leads with precordial R/S transition in V4, which was typical features of idiopathic VT arising from the RVOT. It is commonly believed that patients with RVOT-VT rarely suffer from underlying structural heart disease, and the prognosis is satisfactory. Because of no abnormality detected by the first UCG screening, the diagnosis of idiopathic RVOT-VT was made even when ARVC was highly suspected in the presence of $\mathrm{T}$ wave inversion in V1-V4 leads, which were characteristic of ARVC. Because RVOT-VT can be adrenergically mediated, $\beta$-receptor antagonist, metoprolol (25 mg, twice daily), was orally administrated, however which could not effectively prevent recurrence of VT. Some studies have suggested that patients with ARVC could manifest the same VT pattern as idiopathic RVOT-VT [3] and $\mathrm{T}$ wave inversion (TWI) in V1-V3 of sinus rhythm, which has a high sensitivity and specificity in the discrimination of ARVC from RVOT [4]. Discrimination between these 2 entities is critical due to different prognoses and therapeutic options. TWI in V1-V3 has been reported in $36 \%$ to $96 \%$ of ARVC patients [5] and has been proposed as a major criterion for the diagnosis of ARVC [6].

UCG is commonly used as a screening tool for the diagnosis of ARVC, and most affected areas of RV involve the apex, the outflow tract and posterior wall, which are known as "triangle of dysplasia" and can be detected by UCG. In this case, twice UCG scan revealed structural and functional normal heart, leading to the misdiagnosis of idiopathic RVOT-VT. Focal minor abnormality within the right ventricle in the early stage of ARVC not detected by UCG could be the major reason, which limited the accuracy and utility of UCG. Compared with UCG, 
CMR has been proved to more precisely assess the regional, morphological abnormalities and potentially the presence of fatty infiltration. The patient referred to CMR imaging and was found localized aneurysm and dilation of the right ventricular free wall, which supported the diagnosis of ARVC. For patients with possible ARVC who do not meet criteria of TFC, CMR may reveal the typical features and alter the subsequent treatment $[7,8]$. It was reported that TWI of right precordial leads usually occurred in patients with extensive right ventricular involvement [9], however, the soldier has pronounced TWI in the setting of minor abnormality, which implies repolarization abnormality and may not be parallel to anatomical changes.

ICD has been proved to be the most effective treatment of ARVC by far. During the follow-up, we observed that the soldier underwent anti-tachycardia pacing (ATP) and shock therapy of ICD in the setting of VT, which successfully terminated tachyarrhymia and prevented it from degenerating into ventricular fibrillation.

\section{REFERENCES}

[1] Dimitrios, A., Nikos P, Angeliki, A., et al. (2011) Arrhythmogenic right ventricular cardiomyopathy/dysplasia. The Hellenic Journal of Cardiology, 52, 452-461

[2] McKenna, W.J., Thiene, G., Nava, A., et al., Task Force of the Working Group Myocardial and Pericardial Dis- ease of the European Society of Cardiology and of the Scientific Council on Cardiomyopathies of the Interna- tional Society and Federation of Cardiology (1994) Di- agnosis of arrhythmogenic right ventricular dysplasia/ cardiomyopathy. British Heart Journal, 71, 215-218. http://dx.doi.org/10.1136/hrt.71.3.215

[3] O’Donnell, D., Cox, D., Bourke, J., et al. (2003) Clinical and electrophysiological differences between patients with arrhythmogenic right ventricular dysplasia and right ventricular outflow tract tachycardia. European Heart Journal, 24, 801-810. http://dx.doi.org/10.1016/S0195-668X(02)00654-1

[4] Daniel, P., Morin, M., Andreas, C., et al. (2010) Usefulness of precordial T-Wave inversion to distinguish arrhythmogenic right ventricular cardiomyopathy from idiopathic ventricular tachycardia arising from the right ventricular outflow tract. American Journal of Cardiology, 105, 1821-1824. http://dx.doi.org/10.1016/j.amjcard.2010.01.365

[5] Nasir, K., Bomma, C., Tandri, H., et al. (2004) Electrocardiographic features of arrhythmogenic right ventricular dysplasia/cardiomyopathy according to disease severity: A need to broaden diagnostic criteria. Circulation, 110, 1527-1534. http://dx.doi.org/10.1161/01.CIR.0000142293.60725.18

[6] Marcus, F., McKenna, W., Sherrill, D., et al. (2009) Diagnosis of arrhythmogenic right ventricular cardiomyopathy/dysplasia (ARVC/D): Proposed modification of the task force criteria. Circulation, in Press.

[7] Khang, L., Colin, E., Hamish, H., et al. (2012) Utility of cardiac magnetic resonance in the evaluation of unselected patients with possible arrhythmogenic right ventricular cardiomyopathy. Clinical Medicine Insights: Cardiology, 6, 153-162.

[8] Giovanni, Q., Syed, I., Andrew, S., et al. (2013) Arrhythmogenic right ventricular cardiomyopathy mimics: role of cardiovascular magnetic resonance. Journal of Cardiovascular Magnetic Resonance, 15, 16. http://dx.doi.org/10.1186/1532-429X-15-16

[9] Nasir, K., Bomma, C., Tandri, H., et al. (2004) Electrocardiographic features of arrhythmogenic right ventricular dysplasia/cardiomyopathy according to disease severity: A need to broaden diagnostic criteria. Circulation, 110, 1527-1534.

http://dx.doi.org/10.1161/01.CIR.0000142293.60725.18 